\title{
Utilisation des plantes indigènes à effet insecticide pour la protection des denrées stockées contre des insectes ravageurs à Boukoko (Centrafrique)
}

\author{
Aba L. TOUMNOU ${ }^{1,2,4,5^{*}}$, D. SECK ${ }^{2}$, S. NAMKOSSERENA ${ }^{3}$, N. CISSE ${ }^{4}$, \\ N. KANDIOURA ${ }^{1}$ et M. SEMBENE ${ }^{1}$ \\ ${ }^{1}$ Université Cheikh Anta Diop de Dakar, Faculté des Sciences etTechnologie, Dakar, Sénégal. \\ ${ }^{2}$ Centre Régionale de Recherche en Écotoxicologie et Sécurité Environnementale (CERES-Locustox), \\ Dakar, Senegal. \\ ${ }^{3}$ Institut Centrafricain de la Recherche Agronomique (ICRA), Centrafrique. \\ ${ }^{4}$ Centre d'Étude Régionale pour l'Amélioration et l'Adaptation à la Sécheresse (CERAAS), Thiès, Sénégal. \\ ${ }^{5}$ Université de Bangui, Faculté des Sciences, Laboratoire des Sciences Agronomiques pour le Développement \\ (LASBAD), Bangui, Centrafrique. \\ *Auteur correspondant, E-mail : toumnou@yahoo.fr Tél.00221 339514993
}

\section{RESUME}

Les paysans utilisent couramment des plantes indigènes pour protéger les denrées des attaques des ravageurs en Centrafrique. Des enquêtes ethnobotaniques effectuées auprès de 79 paysans centrafricains à Boukoko ont permis de collectionner 9 plantes indigènes à effet insecticide potentiel. Les données d'enquêtes ethnobotaniques ont été traitées par une analyse factorielle de correspondance dont les deux premiers axes expliquent $84,75 \%$ de la variance totale. Ces plantes se repartissent en 7 familles dont les Méliacées et les Huacées sont les plus utilisées; les écorces étant les parties les plus exploitées. Le dispositif utilisé pour les tests biologiques à l'égard de Sitophilus zeamaïs et Tribolium castaneum est un bloc complètement randomisé. Les variables mesurées sont le taux de mortalité et le nombre d'insectes émergés. Ces variables sont soumises à une analyse de variance modèle fixe à 4 facteurs dans Minitab 14. Les résultats ont montré qu'Afrostyrax lepidophyllus et Trichilia gilgiana sont les espèces les plus prometteuses. Le logiciel RIZA a été utilisé pour calculer les CL50. Trichilia gilgiana appliqué à Sitophilus zeamaïs et Tribolium castaneum après 25 jours de traitement a présenté respectivement une CL50 de $5,13 \mathrm{~g} / 100 \mathrm{~g}$ et de $5,30 \mathrm{~g} / 100 \mathrm{~g}$ de spéculations tandis qu'Afrostyrax lepidophyllus a présenté une CL50 de 5,60 g/100g et de CL 5,86 g/100g.

(C) 2012 International Formulae Group. All rights reserved.

Mots clés : Plantes indigènes, protection, denrées stockées, insectes ravageurs.

\section{INTRODUCTION}

En Afrique subsaharienne, la sécurité alimentaire et nutritionnelle des populations est un enjeu majeur. La sous-alimentation touche plus du tiers des habitants (plus de 200 millions de personnes) et la dénutrition chronique concerne près de $40 \%$ des enfants de moins de 5 ans (OMS, 2011). Les conséquences de l'insécurité alimentaire et nutritionnelle sont dramatiques. Les malnutritions sont responsables de 35 à $55 \%$ des décès d'enfants de moins de 5 ans, de $20 \%$ de la mortalité maternelle, de $11 \%$ de la morbidité globale (OMS, 2011). La perte de 
capital humain et économique est estimée à $10 \%$ des gains sur une vie, à plusieurs points de PIB au niveau des pays (WSP, 2012). La pression démographique et la hausse des prix des produits alimentaires qui se poursuivent, pèsent toujours sur le pouvoir d'achat des consommateurs en Afrique subsaharienne (Benz et al., 2010 ; Fao, 2008). En dépit des flambées du prix des denrées alimentaires, les pertes dues aux insectes nuisibles en Afrique est de l'ordre de 25 à $30 \%$ (Gueye et al., 2011 ); autrement dit presque le tiers de ce qui est produit ne parvient pas aux consommateurs. Ces pertes participent à creuser l'insécurité alimentaire et à accentuer la pauvreté en favorisant le recours massif à des importations de denrées alimentaires (Benz et al., 2010). De nombreuses solutions techniques ont été développées par la recherche pour améliorer l'efficacité de la gestion des insectes ravageurs des cultures et des denrées stockées (lutte chimique, la lutte biologique, résistance variétale) mais leur appropriation est restée limitée. La lutte chimique atteint par ailleurs certaines limites avec son impact significatif sur l'environnement ( Khaliq et al., 2007 ; OMS, 2008 ; Ramezani et al., 2008; Regnault, 2008 ; Travis et al., 2009 ; Fianko et al., 2011 ) et le développement de résistances aux insecticides par certains ravageurs (Sayyed et al., 2008; Schuster et Smeda, 2007). Ces éléments nécessitent d'explorer des solutions techniques alternatives comme le développement des nouvelles molécules d'origine végétale. L'utilisation des substances végétales en tant que biopesticides dans la protection des grains au cours du stockage contre les insectes a fait l'objet de nombreux travaux notamment en zone tropicale (Foua-Bi, 1993; Seck, 1996 ; Regnault et al., 2008; Gueye et al., 2011 ; Raymond et al., 2011; Konno, 2011; Aba Toumnou et al., 2012). Il apparaît un réel potentiel de certaines espèces végétales dont l'efficacité insecticide a été démontrée.
L'objectif de cette étude était d'identifier les plantes indigènes utilisées dans le milieu paysan de Boukoko pour la lutte contre les ravageurs des denrées stockées et d'évaluer l'efficacité des poudres des écorces du tronc de ces plantes à l'égard de Sitophilus zeamaïs et Tribolium castaneum afin d'identifier les plantes les plus prometteuses.

\section{MATERIEL ET METHODES}

Choix du site d'enquêtes ethnobotaniques

La Commune de Boukoko se trouve en marge septentrionale du bassin forestier congolais (Figure 1) et elle reçoit une moyenne pluviométrique de plus de $1600 \mathrm{~mm}$ de pluie par an; ce qui est favorable à l'agriculture. C'est une zone de forte diversité agricole (bananes douces et plantain, manioc, arachide, niébé, taro, ignames, maïs...). Les Mbati ou Issongo et les NGbaka se sont installés après les pygmées Aka qui sont les autochtones de Boukoko (Decoudras, 1984). Peu d'études ont été réalisées sur les espèces forestières à effet biopesticide potentiel utilisées pour la protection des récoltes à Boukoko.

\section{Méthode de collecte des données, identification et séchage des échantillons des plantes}

Avant d'explorer des ressources forestières, la carte et autres informations générales de la Commune de Boukoko ont été obtenues auprès de la Mairie de cette Commune. La Commune a été divisée en différents sites et des visites ont été effectuées dans chaque site en Mars et Avril 2011. Un questionnaire a été préparé et utilisé comme un outil pour la collecte d'informations. Le questionnaire a été divisé en deux parties: la démographie et les données ethnobotaniques. La première partie comprenait des données démographiques, nom de l'emplacement, l'âge, l'éducation et la profession. La deuxième partie comprenait des questions sur 
le mode de stockage des denrées alimentaires post-récolte, les essences forestières utilisées pour la protection des denrées, le mode d'utilisation de ces essences et la durée de protection et de stockage. En règle générale, les 79 répondants étaient des personnes âgées dont la tranche d'âge variait de 35 à 70 ans. Les échantillons des plantes sont récoltés dans leur milieu naturel. Sur la base des noms locaux ; des herbiers sont montés, des plantes photographiées et des échantillons récoltés. Les noms scientifiques ont été identifiés par les herboristes du Ministère des Eaux et Forêts de Centrafrique et les Enseignants Chercheurs de l'Université de Bangui. La flore illustrée de Centrafrique (Tisserant, 1950) a été utilisée pour la confirmation des noms des espèces. Les écorces des plantes récoltées sont séchées à l'ombre pendant deux semaines avant d'être transformées en poudre pour les expériences.

\section{Choix des insectes et élevage de masse}

Sitophilus zeamaïs et Tribolium castaneum sont des ravageurs très actifs dans les systèmes paysans de stockage des récoltes d'après les paysans de la Commune de Boukoko. Ces deux insectes sont récoltés au champ et élevés respectivement sur maïs et arachide au Laboratoire d'Entomologie à l'ICRA (Institut Centrafricain de Recherche Agronomique). L'élevage de masse est réalisé à la température de $28{ }^{\circ} \mathrm{C}$ et une humidité relative de $70 \pm 5 \%$. 150 insectes de sexe confondu sont mis en élevage sur $250 \mathrm{~g}$ de maïs et arachide dans les bocaux en verre borosilicaté perforés et recouverts de toile de moustiquaire de façon à permettre aux insectes de respirer. Une semaine après avoir assuré la ponte, les adultes ont été éliminés ; les œufs pondus ont évolué jusqu'à donner des adultes de la première génération 35 jours après l'infestation. Ces adultes de la première génération sont mis en élevage dans les mêmes conditions que sus-citées. Une semaine après avoir assuré la ponte, les adultes ont été éliminés ; les œufs pondus ont évolué jusqu'à donner des adultes de la deuxième génération qui sont utilisés pour les tests biologiques.

\section{Traitements statistiques des données}

Pour les données d'enquêtes ethnobotaniques, une analyse factorielle de correspondance à l'aide du logiciel Minitab 14 a été utilisée pour identifier les espèces utilisées pour la protection des denrées citées. Des diagrammes ont été aussi construits pour évaluer l'abondance spécifique par famille et les parties des plantes utilisées. Pour les données des tests biologiques avec les poudres des plantes, les variables mesurées sont le taux de mortalité, le nombre d'insectes survivants et le nombre d'insectes émergés. La mortalité calculée a été obtenue en appliquant la formule d'Aboth (1925): Mc = $(\mathrm{Mo}-\mathrm{Mt}) /(100-\mathrm{Mt}) * 100$; (où Mo = mortalité dans les lots traités, Mt = mortalité dans le témoin et $\mathrm{Mc}=$ mortalité calculée). Les variables taux de mortalité, nombre des insectes survivants et nombre des insectes émergés sont soumises à une analyse de variance, modèle fixe à quatre facteurs (plantes, insectes, doses et temps). La variable taux de mortalité a subi une transformation $\arcsin (\mathrm{x}=$ taux de mortalité, $\mathrm{n}=$ taille de la population; $n=1999$ ) afin de normaliser la population et de stabiliser la variance. La méthode General Linear Model dans Minitab 14 a été utilisée pour l'analyse statistique des données collectées. Les variables nombre d'insectes survivants et nombre d'insectes émergés quant à elles ont subi une transformation racine carré afin de normaliser la population et de stabiliser la variance. Les courbes et les tableaux sont utilisés pour présenter le résultat des analyses. 


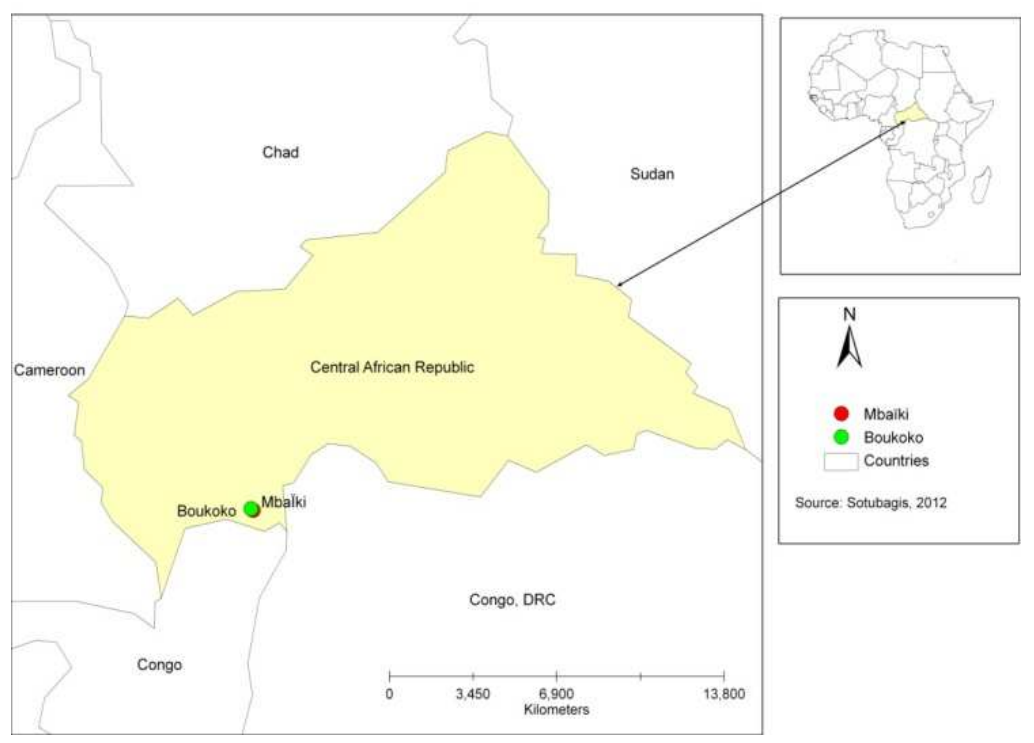

Figure 1: Localisation de la Commune de Boukoko.

\section{RESULTATS}

Espèces utilisées pour conserver les denrées citées

Les valeurs propres des principaux axes extraits de l'analyse factorielle de correspondance indiquent que les 2 premiers facteurs expliquent $84,75 \%$ de l'information de la matrice espèces-denrées protégées (Tableau 1). Le plan factoriel formé par ces deux axes (F1 et F2) permet de définir deux groupes d'utilisations de plantes/maladies (Figure 2). Le groupe 1 est constitué des espèces les plus utilisées pour la protection du maïs, de l'arachide et du niébé. Ce sont : 1 (Tetrorchiduim didymostemon B.), 2 (Croton ambrevillei), 4 (Trichilia gilgiana), 5 (Justicia verticillaris L.), 6 (Capsicum frutescens) et 8 (Khaya senegalensis). Le groupe 2 est constitué des espèces les plus utilisées pour la protection de la cosette de manioc. Ce sont : 3 (Copaifera milbdraedii H.), 7 (Afrostyrax lepidophyllus Mildbr) et 9 (Strophanthus gratus).

\section{Abondance des espèces par famille des plantes utilisées pour la protection des denrées citées}

Les espèces indigènes utilisées pour la protection des denrées citées sont réparties en
7 familles (Figure 3). Les plantes les plus utilisées sont de la famille des Méliacées (89 citations), Huacées (71 citations) et Euphorbiacées (63 citations). Les espèces moyennement utilisées appartiennent aux familles des Solanacées (42 citations) et des Apocynacées (38 citations). Les espèces les moins citées appartiennent aux familles des Fabacées (12 citations) et des Acanthacées (14 citations).

\section{Parties utilisées}

- Les écorces du tronc des plantes sont plus utilisées pour la protection des denrées citées.

- Les feuilles associées aux écorces du tronc des plantes sont moyennement utilisées.

- $\quad$ Les fruits sont moins utilisés (Figure 4).

\section{Tests biologiques}

Les résultats d'analyse de la variance relatifs à l'effet insecticide des poudres de plantes sur Sitophilus zeamaïs et Tribolium castaneum révèlent que le taux de mortalité a une variation très hautement significative suivant la dose, l'insecte et le temps considéré d'une part, selon la plante, l'insecte et le temps d'autre part $(\mathrm{P}<0,001)$. Par ailleurs, seul l'effet de l'interaction plante 
dose insectes n'est pas significative $(\mathrm{P}>0,05)$, cela implique que l'effet insecticide observé dépend de la nature de la plante, de la dose et de l'insecte.

Les Figures 7 et 8 montrent l'allure de la courbe des insectes émergés en fonction des doses appliquées, des insectes et du temps. Afrostyrax lepidophyllus Mildbr assure une bonne protection du milieu traité aux doses 3, 4 et 5. Tetrorchiduim didymostemon et Croton ambrevillei présentent aussi un effet protecteur du milieu traité aux doses 3, 4 et 5 . (Figures 7 et 8 ).
Les Figures 5 et 6 montrent l'évolution du traitement en fonction de la dose, du temps et des insectes. Les plantes prometteuses sont Afrostyrax lepidophyllus et Trichilia gilgiana. Sitophilus zeamaïs et Tribolium castaneum présentent une même sensibilité pour les doses 4 et 5 . La courbe de mortalité en fonction du temps montre qu'à T5, T10 et T25 le taux de mortalité est élevé, cela implique que les poudres de plante agissent à long terme (Figure 5).

Tableau 1: Valeurs propres et pourcentage de variance.

\begin{tabular}{lccc}
\hline Valeurs propres et pourcentage de variance & F1 & F2 & F3 \\
\hline Valeurs propres & 0,3881 & 0,1509 & 0,1046 \\
\% variance & 0,6030 & 0,2345 & 0,1625 \\
$\%$ cumulé & 0,6030 & 0,8375 & 1,000 \\
\hline
\end{tabular}

Tableau 2: Résultat d'analyse de variance sur les paramètres observés.

\begin{tabular}{lccccc}
\hline Source de variation & DL & \multicolumn{2}{c}{ Mortalité } & \multicolumn{2}{c}{ Insectes émergés } \\
\hline Plante & 7 & $\mathbf{F}$ & $\mathbf{P}$ & $\mathbf{F}$ & $\mathbf{P}$ \\
dose & 4 & 155,59 & 0,000 & 5,04 & 0,000 \\
Insectes & 290,21 & 0,000 & 15,37 & 0,000 \\
Temps & 6 & 12,01 & 0,001 & 11,97 & 0,001 \\
Plante dose & 28 & 93,54 & 0,000 & 43,76 & 0,000 \\
Plante insectes & 7 & 21,24 & 0,000 & 0,96 & 0,529 \\
Plante temps & 42 & 4,19 & 0,000 & 2,85 & 0,006 \\
Dose insectes & 4 & 2,51 & 0,000 & 1,14 & 0,283 \\
Dose temps & 24 & 4,99 & 0,001 & 1,43 & 0,220 \\
Insectes temps & 6 & 2,97 & 0,000 & 4,27 & 0,000 \\
Plante dose insectes & 28 & 85,19 & 0,000 & 8,92 & 0,000 \\
Dose insectes temps & 24 & 1,37 & 0,096 & 0,85 & 0,693 \\
Plante insecte temps & 42 & 3,94 & 0,000 & 0,91 & 0,563 \\
Erreur & 3,81 & 0,000 & 1,46 & 0,056 \\
Total & 2576 & & & & \\
\hline
\end{tabular}




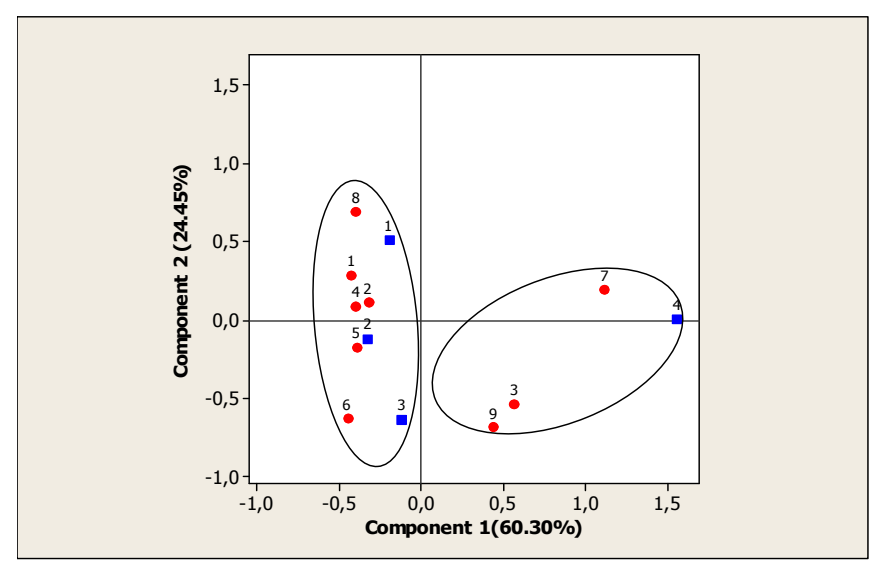

Figure 2: Plantes/denrées protégées par l'analyse factorielle des correspondances (AFC). $e 1_{=}$ Tetrorchiduim didymostemon B., e2 =Croton ambrevillei , e3 =Copaifera milbdraedii $\mathrm{H}_{\mathrm{H}}$ e $4=$ Trichilia gilgiana, e $5_{=}$Justicia verticillaris $L$., e6=Capsicum frutescens,$e 7=$ Afrostyrax lepidophyllus Mildbr, $e 8_{=}$Khaya senegalensis, $e 9=$ Strophanthus gratus, $1=$ Maïs, 2 = Arachide, $3=$ Niébé, $4=$ Cosette manioc.

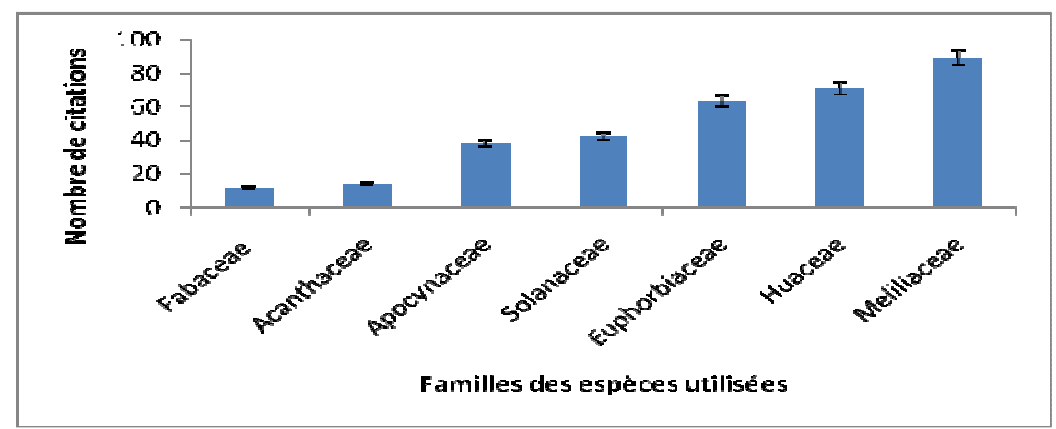

Figure 3: Abondance des espèces citées par famille.

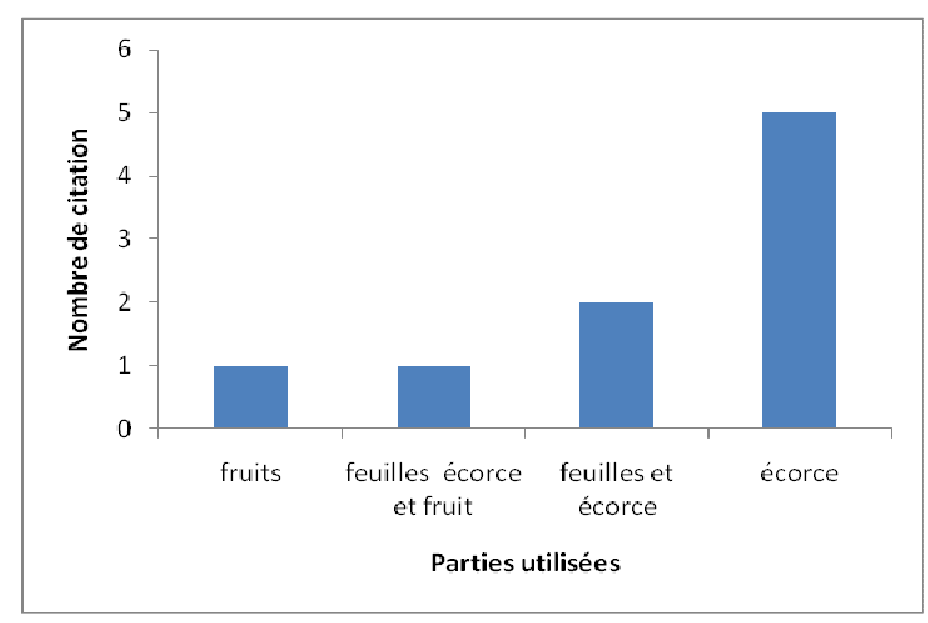

Figure 4: Différentes parties des plantes utilisées pour la protection des denrées stockées. 


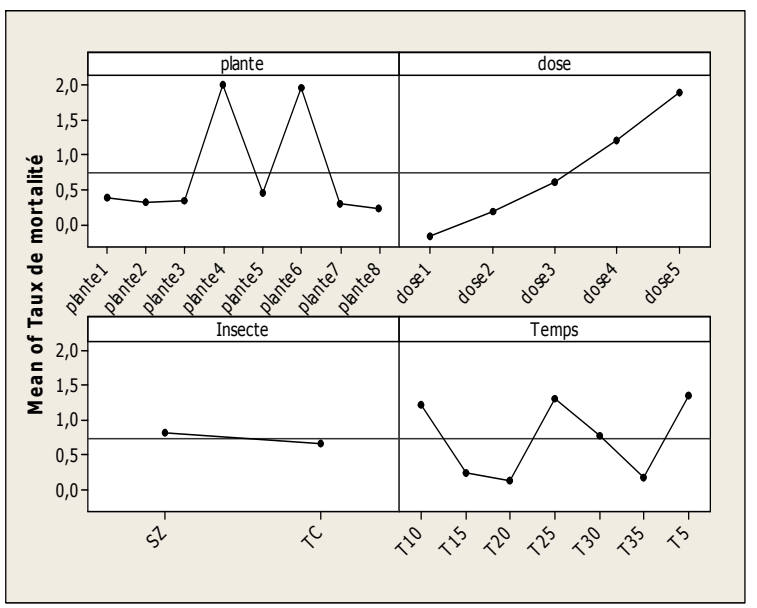

Figure 5: Courbe de mortalité en fonction du temps.

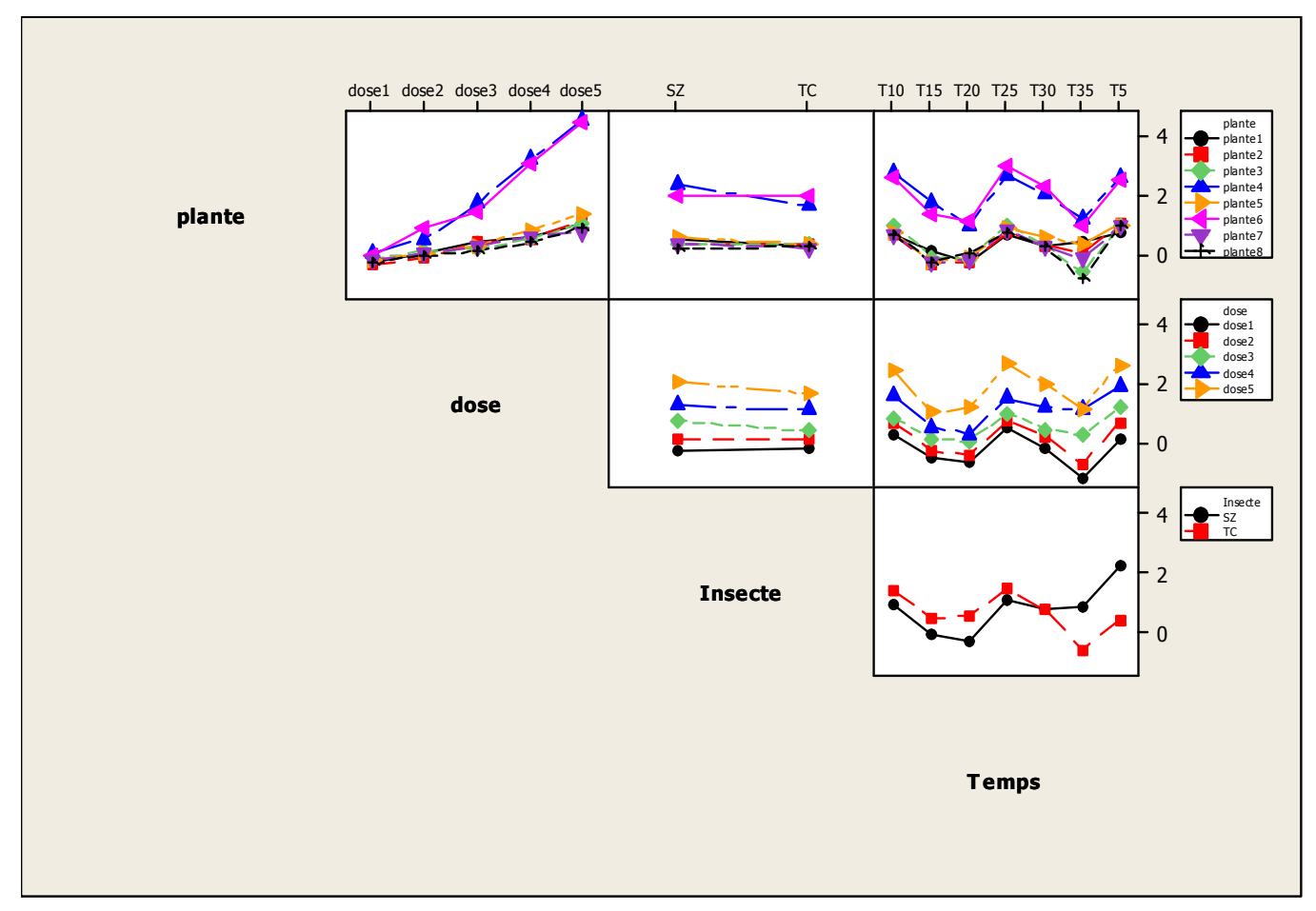

Figure 6: Interactions entre plante, dose et insectes. Plante1 = Tetrorchiduim didymostemon B., plante2=Croton ambreville , plante $3=$ Copaifera milbdraedii $H$., plante4= Trichilia gilgiana, plante5= Justicia verticillaris $L$., plante6= Afrostyrax lepidophyllus Mildbr, plante7= Khaya senegalensis, plante8=Strophanthus gratus, $\mathrm{SZ}=$ Sitophilus zeamaïs, TC= Tribolium castaneum, dose $1=1 \mathrm{~g}$, dose $2=2 \mathrm{~g}$, dose $3=3 \mathrm{~g}$, dose $4=4 \mathrm{~g}$ et dose $5=5 \mathrm{~g}, \mathrm{~T} 5=5$ jours de traitement, $\mathrm{T} 10=10$ jours de traitement, $\mathrm{T} 15=15$ jours de traitement, $\mathrm{T} 20=20$ jours de traitement, $\mathrm{T} 25=25$ jours de traitement, $\mathrm{T} 30=30$ jours de traitement, $\mathrm{T} 35=35$ jours de traitement. 


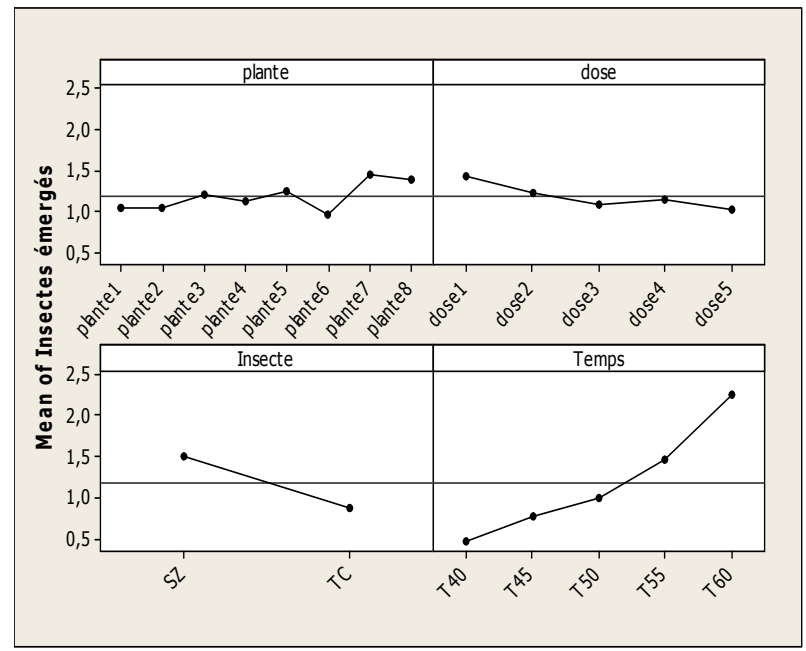

Figure 7: Courbe d'insectes émergés en fonction des doses, d'insectes et du temps.

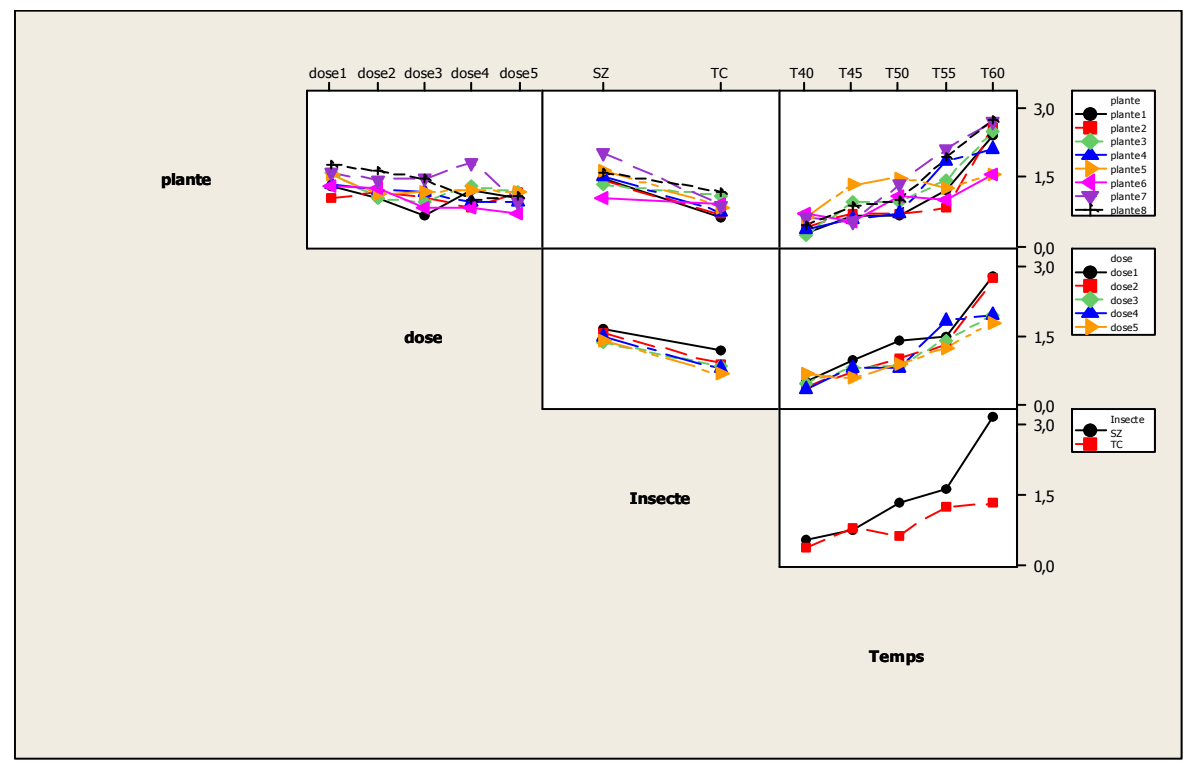

Figure 8: Interaction entre plantes, doses et insectes émergés en fonction du temps. $\mathrm{T} 40=40$ jours, $\mathrm{T} 45=45$ jours, $\mathrm{T} 50=50$ jours, $\mathrm{T} 55=55$ jours et $\mathrm{T} 60=60$ jours.

\section{DISCUSSION}

D'après les analyses de nos données, les plantes les plus citées pour la lutte contre les insectes ravageurs appartiennent à la famille des Méliacées. Les méliacées ont fait l'objet des études approfondies pour leur propriété insecticide. L'activité insecticide de
l'Azadirachtine extraite de l'Azadirachta indica qui est une méliacée a été prouvée (Isman et al., 1990). Au Sénégal, les plantes de la famille des méliacées sont beaucoup utilisées dans la lutte contre les insectes ravageurs des grains des céréales et légumineuses post-récolte (Aba Toumnou et 
al., 2012). Les tests de l'activité insecticide effectués avec les poudres séchées de Trichilia gilgiana ont montré une efficacité sur Sitophilus zeamaïs et Tribolium castaneum (Figures 6 et 7). Trichilia gilgiana est une méliacée. Les analyses chimiques d'une autre espèce du genre Trichilia, Trichilia martiana ont confirmé la présence de molécules à effet insecticide potentiel. Trichilia gilgiana utilisée pour la protection des denrées stockées à Boukoko pourrait renfermer aussi des métabolites secondaires à effet insecticide potentiel. Les plantes identifiées sont des Angiospermes. Les Angiospermes contiennent des alcaloïdes qui sont des métabolites secondaires constitués des atomes d'azote secondaire, tertiaire ou quaternaire dans leurs structures (Bruneton, 2009). Ils sont métaboliquement actifs et jouent un rôle important dans la physiologie des plantes ou des organismes. Les alcaloïdes possèdent des propriétés répulsives ou anti appétantes à l'égard des insectes ravageurs (Pelletier, 2001). Le latex de la famille des Euphorbiacées est constitué des alcaloïdes, des diterpènes et des triterpènes (Bruneton, 2009). Les diterpènes et les triterpènes possèdent des propriétés insecticides et insectifuges (Ngamo et Hance, 2007 ; Bruneton, 2009; Raymond et al., 2011; Konno, 2011). Les espèces de la famille des Euphorbiacées telles que Tetrorchiduim didymostemon $B$. et Croton ambrevillei moyennement citées par les paysans lors des enquêtes ethnobotaniques pour la lutte contre les insectes ravageurs en post-récolte pourraient renfermer des substances à effet insecticide, insectifuge ou anti appétant.

Tetrorchiduim didymostemon B., Croton ambrevillei , Copaifera milbdraedii H., Trichilia gilgiana , Justicia verticillaris L., Afrostyrax lepidophyllus Mildbr, Khaya senegalensis et Strophanthus gratus sont des plantes supérieurs (Bedel et al., 1998). Les plantes supérieures produisent largement des tanins (Raymond et al., 2011; Konno, 2011). Les tanins présentent un effet direct toxique pour certaines espèces d'insectes (Raymond et al., 2011). Les tanins influencent sur la croissance, le développement et la fécondité de plusieurs insectes ravageurs (Vandenborre et al., 2011). La croissance réduite causée par les tanins a des inconvénients majeurs pour l'insecte, avec un plus faible nombre d'œufs et une plus petite taille d'œufs, ce qui affecterait la survie et la santé des individus de la génération subséquente (Meric, 2005).

Les graines d'A. lepidophyllus sont utilisées comme épice alimentaire par les femmes tandis que les écorces du tronc ainsi que les feuilles sont utilisées par les tradipraticiens dans la pharmacopée locale à Boukoko. Ngono et al. (2006) ont mis en évidence la présence d'Afrostyraxthioside A, d'Afrostyraxthioside B et d'Afrostyraxthioside $\mathrm{C}$ dans A. lepidophyllus. Ces molécules font partie des composés soufrés. L'activité insecticide ou insectifuge des composés soufrés de certaines plantes comme Cinéol, Menthol, Thymol, Ail, giroflier, Citronelle , Géranum, Eucalyptus, Chenopodium ambrosioides, Agrunes, Romarin et Canelle sont connues (Chiasson et Beloin, 2007). Afrostyraxthioside A, Afrostyraxthioside B et Afrostyraxthioside C composés soufrés d'A. lepidophyllus pourraient être aussi source de molécules insecticides ou insectifuges.

\section{Conclusion}

Les tests biologiques effectués avec les poudres séchées des écorces du tronc des 8 plantes indigènes ont permis d'identifier 2 plantes prometteuses (Trichilia gilgiana et Afrostyrax lepidophyllus). Ces 2 plantes font l'objet des études approfondies (tests biologiques avec les extraits aux solvants organiques et d'analyse chimique) afin de caractériser les molécules susceptibles de l'activité insecticide observée avec les poudres séchées.

\section{REMERCIEMENTS}

Les auteurs remercient le CERAAS (Centre d'Etude Régional pour l'Amélioration de l'Adaptation à la Sécheresse) et l'ICRA (Institut Centrafricain de la Recherche Agronomique) respectivement pour leurs 
appuis financier et logistique qui ont permis de réaliser les prospections auprès des paysans et d'effectuer les tests biologiques préliminaires. Nos sincères remerciements aussi à DAAD (Deutscher Akademischer Austausch Dienst) pour l'octroi d'une bourse d'étude.

\section{RÉFÉRENCES}

Aba Toumnou L, Seck D, Thiaw C, Cisse N, Kandioura N, Sembene M. 2012. Farmers' pesticidal plant use in the protection of stored cereal and legume grains:ethnobotanical surveys in some rural communities in Senegal. International Journal of Science and Advanced Technology, 2(3): 25-33.

Abbott WS. 1925. A method of computing the effectiveness of an insecticide. Journal of Economic Entomology, 18(2): 265-267.

Bedel F, Durrieu de Madron L, Dupuy B, Favrichon V, Bar-hen A, Narboni P. 1998. Dynamique de croissance dans des peuplements exploités et éclaircis de forêt dense africaine. Dispositif de M'baiki en République Centrafricaine 1982-1995. FORAFRI, CIRAD-Forêt.

Benz DH, Diallo A, Lançon F, Meuriot V, Rasolofo P, Temple L, Wane A. 2010. L'Imparfaite Transmission des Prix Mondiaux aux Marchés Agricoles d'Afrique Subsaharienne. Farm, Cirad; $28 \mathrm{p}$.

Bruneton J. 1993. Pharmacognosie, Phytochimie, Plantes Médicinales $\left(2^{\mathrm{e}}\right.$ edn). Tec \& Doc: Paris; 914 p.

Bruneton J. 2009. PharmacognosiePhytochimie, Plantes Médicinales ( $4^{\mathrm{e}}$ édn, revue et augmentée). Tec \& Doc: Paris; $1288 \mathrm{p}$.

Chiasson H, Beloin N. 2007. Les huiles essentielles, des biopesticides «nouveau genre ». Bulletin de la Société d'Entomologie du Québec, 14(1): 23.

Decoudras P-M. 1984. Industries et artisanat. In Atlas de la République Centrafricaine, Vennetier P, Laclavère G (éds). Editions Jeune-Afrique: Paris; 49 p.
FAO. 2008. Perspectives de récolte et situation alimentaire. Système mondial d'information et d'alerte rapide sur l'alimentation et l'agriculture, $\mathrm{N}^{\circ} 4$.

Fianko JR, Donkor A, Lowor ST, Yeboah PO, Glover ET, Adom T, Faanu A. 2011. Health risk associated with pesticide contamination of fish from the Densu River Basin in Ghana. Journal of Environmental Protection, 2(2): 115-123.

Gueye MT, Seck D, Ba S, Hell K, Sembène M, Wathelet J-P, Lognay G. 2011. Insecticidal activity of Boscia senegalensis (Pers.) Lam ex Poir. on Caryedon serratus (Ol.) pest of stored groundnuts. African Journal of Agricultural Research, 30(6): 6348-6353.

Isman MB, Koul O, Luczynski A, Kaminskis J. 1990. Insecticidal and antifeedant bioactivities of neem oils and their relationship to azadirachtin content. $J$. Agric. Food Chem., 38: 1406-1411.

Khaliq A, Attique MNR, Sayyed AH. 2007. Evidences for resistance to organophosphates and pyrethroids in Plutella xylostella (Lepidoptera: Plutellidae) from Pakistan. Bull. Entomol. Res., 97: 191-200.

Konno K. 2011. Plant latex and other exudates as plant defense systems: Roles of various defense chemicals and proteins contained therein. Phytochemistry, 72(13): 15101530.

Meric K. 2005. Études sur les composés polyphénoliques en relation avec l'alimentation de la tordeuse des bourgeons de l'épinette (Choristoneura fumiferana (Clem.). Thése de Doctorat, Sciences Forestières, Univ. Laval.

Ngamo LST, Hance T. 2007. Diversité des ravageurs des denrées et méthodes alternatives de lutte en milieu tropical. Tropicultura, 25(4): 215-220.

Ngono NA, Lavault M, Seraphin D, Landreau A, Richomme P. 2006. Three 1-thio- $\beta$-Dglucopyranosides from the seeds of Afrostyrax lepidophyllus Mildbr. Elsevier, 341(17): 2799-2802. 
Organisation Mondiale de la Santé (OMS). 2011. Rapport sur la malnutition en Afrique subsharienne. Bull. N5. 52p.

Pelletier SW. 2001. Alkaloids : Chemical and Biological Perspectives. University of Georgia: USA; 656p.

Ramezani M, Oliver DP, Kookana RS, Gurjeet G, Preston C. 2008. Abiotic degradation (photodegradation and hydrolysis) of imidazolinone herbicides. J. Environ. Sci. Health. Part B, 43: 105112.

Raymond V, Barbehenn C, Constabel P. 2011. Tannins in plant-herbivore interactions. Phytochemistry, 72: 1551-1565.

Regnault RC, Philogène BJR, Vincent C. 2008. Biopesticides d'Origine Végétale ( $2^{\text {ème }}$ édn). Lavoisier: Paris; 550p.

Sayyed AH, Ahmad M, Crickmore N. 2008. Fitness costs limit the development of resistance to indoxacarb and deltamethrin in Heliothis virescens (Lepidoptera: Noctuidae). J. Econ. Entomol., 101: 1927-1933.

Schuster CL, Smeda RJ. 2007. Management of Amaranthus rudis $S$. in glyphosate resistant corn (Zea mays L) and soybean (Glycine max L. Merr.). Crop Prot., 26: 1436-1443.

Tisserant C. 1950. Catalogue de la flore de l'Oubangui-Chari. Mémoire de l'Institut d'Etudes Centrafricaines, Brazzaville, 2, p. 165.

Travis R, Legleiter K, Bradley W. 2009. Evaluation of herbicide programs for the management of glyphosate-resistant waterhemp (Amaranthus rudis) in Maize. Crop Protection, 28: 917-922.

Vandenborre G, Smagghe G, Van Dammea JM. 2011. Plant lectins as defense proteins against phytophagous insects. Phytochemistry, 72: 1538-1550.

Wheeler DA. 2000. The effet of Trichilia Americana extract on the growth development and behavior of the Asian army worm, Spodoptera litura. $\mathrm{PhD}$ Thesis, University of British Columbia.

WSP (Water and Sanitation Program). 2012. Africa: Economics of Sanitation Initiative. [cited 2012 April 18]. Available from www. worldbank.org. 